\title{
LOW-MASS RADIATION-HARD BEAM PROFILE MONITORS FOR HIGH ENERGY PROTONS USING MICROFABRICATED METALTHIN-FILMS
}

\author{
Didier Bouvet ${ }^{1}$, Jacopo Bronuzzi ${ }^{1,2}$, Blerina Gkotse ${ }^{2}$, Georgi Gorine ${ }^{1,2}$, \\ Alessandro Mapelli², Isidre Mateu ${ }^{2}$, Viktoria Meskova ${ }^{2}$, Giuseppe Pezzullo², \\ Federico Ravotti ${ }^{2}$, Jean-Michel Sallese ${ }^{1}$, Ourania Sidiropoulou ${ }^{2}$
}

${ }^{1}$ École Polytechnique Fédérale de Lausanne (EPFL), Lausanne, Switzerland

${ }^{2}$ European Organization for Nuclear Research (CERN), Geneva, Switzerland

\begin{abstract}
In High Energy Physics (HEP) experiments built at the European Organization for Nuclear Research (CERN) it is a common practice to expose electronic components and systems to particle beams, in order to assess their level of radiation tolerance and reliability when operating in a radiation environment. One of the facilities used for such tests is the CERN Proton Irradiation Facility (IRRAD), where several hundreds of samples are irradiated yearly with a $24 \mathrm{GeV} / \mathrm{c}$ proton beam extracted from the CERN Proton Synchrotron (PS) accelerator. In order to properly control the irradiation beam and guarantee reliable results during the tests, Beam Profile Monitor (BPM) devices are used. The current BPMs are fabricated as standard flexible PCBs featuring a matrix of metallic sensing pads. When exposed to the particle beam, secondary electrons are emitted from each pad, thus generating a charge proportional to the particle flux crossing the pads. The charge is measured individually for each pad using a dedicated readout system, and so the shape, the position and the intensity of the beam-spot are obtained. Beam profile determination of high intensity beams implies the usage of non-invasive and radiation tolerant $\left(\sim 10^{18} \mathrm{p} / \mathrm{cm}^{2} /\right.$ year $)$ devices. This study proposes a new fabrication method using standard microfabrication techniques in order to improve the radiation tolerance of the BPMs while greatly reducing the device thickness, thus making them also appropriate to be used for the monitoring of lower energy particle beams.
\end{abstract}

Key words: Irradiation, secondary electron emission, secondary electron yield, beam profile monitor, high energy protons, proton beam, cleanroom, microfabrication, thin films

\section{INTRODUCTION}

In the East Hall Experimental Area of the Proton Synchrotron (PS) accelerator at the European Organization for Nuclear Research (CERN), highenergy and high-intensity particle beams are used. The IRRAD facility, built on the PS-T8 beam-line, is dedicated to perform irradiation experiments of detectors, electronics systems and components with a $24 \mathrm{GeV} / \mathrm{c}$ primary proton beam [1].

The protons are delivered to IRRAD in bursts, or "spills" of $\sim 400$ ms duration, with a typical intensity of $5 \cdot 10^{11} \mathrm{p} / \mathrm{spill}$. During operation, multiple irradiation experiments can be performed simultaneously on 8 motor-controlled tables which are distributed along an approximately 30 meters long beam line. Typically, each table features 3 irradiation positions (labeled left, center and right) which can be remotely positioned across the beam.

In order to ensure that each device under test (DUT) receives the requested cumulated particle fluence, the beam must be correctly aligned on all the tables. For this purpose, a distributed beam monitoring system with multiple measurement points along the beam line is needed. Moreover, the beam position and shape are often affected by changes on the PS extraction parameters. Consequently, to perform the necessary corrections, the beam monitoring must be permanent and real time. This has severe implications for the beam monitoring devices in terms of radiation tolerance since, ideally, they should be capable of enduring particle fluence at least equivalent to the one accumulated during one year of IRRAD operation $\left(\sim 10^{18} \mathrm{p} / \mathrm{cm}^{2}\right)$.

To satisfy these requirements, custom-made Beam Profile Monitors (BPM) are used [2]. They consist of a rectangular-shaped, flexible printed circuit board (PCB), patterned with a matrix of metallic sensing pads on one end, and a multi-pin connector on the opposite one. PCB traces connect electrically the pads to the connector. During operation, the part to the sensing pads is placed directly in the beam and, via Secondary Electron Emission (SEE) [3], a charge proportional to the beam intensity, is generated on each pad. Via the multi-pin connector, the BPM is connected to dedicated readout electronics [4], which measures the amount of charge generated on each pad for each spill, thus resulting in a single beam profile per spill.

The BPMs used during the 2014-2018 IRRAD run are multi-layer flexible PCB. The stack is composed of six copper layers ( $15 \mu \mathrm{m}$-thick) separated by polyimide (80 $\mu \mathrm{m}$-thick) as an insulator substrate. Glue is used in between PCB layers to hold the stack together. BPMs with different layouts were used (Fig. 1): the so-called standard BPM, consisting of a full matrix of 39 pads to measure the full beam profile at 4 fixed locations along the beam line; the mini-BPM, installed on the center

*isidre.mateu@cern.ch 
position of each motorized table for alignment, and which consist of 9 pads distributed in a cross shape, to measure the beam profile projections on the vertical and horizontal axis; finally, the single-pad BPM, used as "in beam monitor", for the lateral positions (right and left) of the tables.

Although this system has proven its usefulness during 4 years of operation, there is room for optimization: first, when all the irradiation tables are inserted in the beam, the sum of all BPMs can add, roughly, $2 \mathrm{~mm}$ of copper and $8 \mathrm{~mm}$ of polyimide to the total material budget in the beam line, which contribute to the scattering of the primary protons and the production of secondary particles; second, several of the BPM detectors suffered from radiation induced damage during the run, presumably due to a failure of the glue between PCB layers which, in some cases, lead to a total loss of the device. Figure 1 shows a standard BPM and three mini-BPMs, all of them presenting signs of damage seen as a "bubble" effect; third, the devices become highly radioactive after prolonged beam exposure, something of special concern when the facility staff needs to replace a broken device.

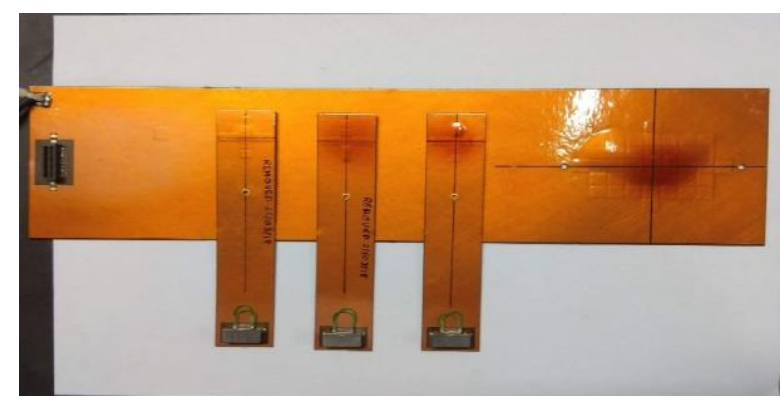

Figure 1. Standard 39-pad BPM (on the back) and three 9-pad mini-BPMs damaged after use. It is possible to observe bubbles and burnt area (in brown), results of radiationinduced damage.

A reduction in the BPM material budget would, therefore, help to minimize the interaction with the beam and, consequently, also their radioactivity and the exposure of the operators. Furthermore, a thinner device would extend the possible usage range towards beams of lower particle energy, such as the ones used in medical applications [5]. The sputtering of very thin metal layers on a passive substrate is investigated in this work as an alternative for the production of the BPM detectors. This technique allows to build much thinner detectors and, as an additional advantage, does not use any glue. Hence, higher radiation resistance is expected. This paper describes the sensing principle, the design and the environment of fabrication, and reports on the experimental results obtained from the irradiation tests on a first batch of prototypes. These new devices are referred in the text as micro-BPMs.

\section{BPM SENSING PRINCIPLE}

When a charged particle beam strikes a metallic surface, two types of secondary electrons are generated: those from the interaction with the surface and those from backscattering in deeper material regions. The SEE process consists of three steps. 1) The target metal absorbs the incident particles and the inner electrons become excited. Some of these electrons receive enough energy to be knocked out from their atoms. The most energetic electrons, or delta rays, can themselves produce secondary ionizations. 2) The secondary electrons diffuse toward the metal surface with energy loss through inelastic collisions. The probability of reaching the surface decreases with the depth at which the secondary electrons are created so, in practice, only electrons generated close to the surface can contribute to the emission (SEE is, then, a surface effect). 3) Finally, some of the secondary electrons can go over the surface potential barrier and escape from it.

A wide range of beam measuring instruments exploiting the SEE principle have been developed in the past [6][7]. Typically, they consist of a vacuum chamber inside which one or more metal foils, placed perpendicular to the beam propagation, are connected to a charge measurement device and used as sensing electrodes. The sensing electrodes are intercalated with biasing electrodes, connected to a positive potential in order to sweep away the emitted electrons. Variations where the foils are replaced by wires also exist [8].

Compared to the instruments referenced above, the BPM concept is simpler, as neither vacuum nor biasing electrodes are used, which makes it a much more compact device that can be easily installed in many locations along the beam line. As a drawback, lower charge generation is expected due to the absence of electric field and the fact of working in air. Although this is not a concern in IRRAD due to the extremely high beam intensity, it could limit its use in other applications. For this reason, one of the main goals of the project is to optimize the BPM design in order to maximize the Secondary Electron Yield (SEY), i.e. how many electrons are emitted per incident particle. Parameters known to affect the SEY are, among others, the emitting metal [9], the particle properties [10], the angle of incidence [11], and surface conditions such as in-situ bake out at high temperature, air exposure, oxide, termination, vacuum... [12]

The BPM sensing layer is required to be made of a low cost, relatively low $\mathrm{Z}$ metal, with short radioactive half-lives after irradiation and available as a target for the sputtering machine. The layer should be made as thin as possible to minimize the scattering of the beam. For the first prototypes, Aluminium (Al) was chosen as the best possible compromise to satisfy these requirements.

\section{DESIGN}

To avoid the complexity of the 39 pad standard $\mathrm{BPM}$, the mini-BPM layout was chosen for the fabrication of the first micro-BPM prototypes. The layout features nine $4 \times 4 \mathrm{~mm}^{2}$ sensing pads, arranged in a cross shape, as shown in Fig. 2. This design allows the monitoring of the beam profile in the two Cartesian directions. For readout purposes, the sensing pads are routed towards contact pads on the opposite end of the device by $100 \mu \mathrm{m}$ wide traces. 


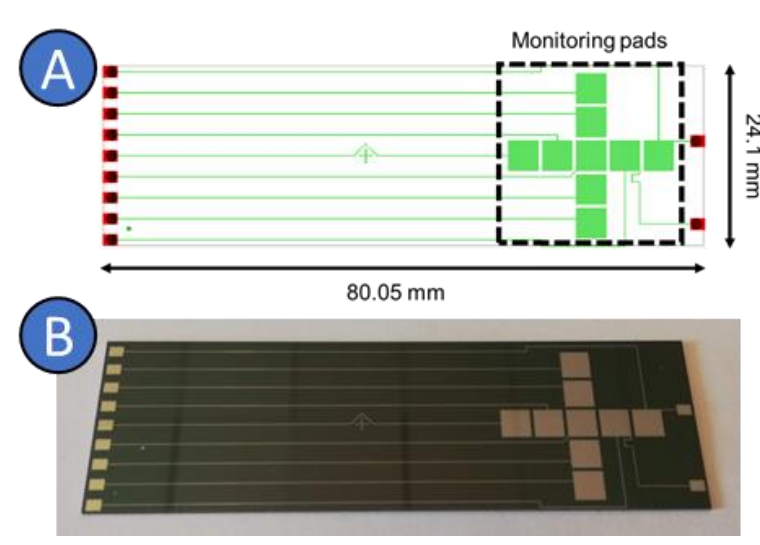

Figure 2. (a) layout of micro-BPMs and (b) image of a fabricated micro-BPM (Si-1X-5Onm). Red squares in (a) represent the contact pads for electrical connections. Monitoring pads for beam profile measurements are disposed in cross, with $4 \mathrm{~mm}$ wide edges.

\section{FABRICATION}

The fabrication of the micro-BPMs was carried out at the Center of Micronanotechnology (CMi) of the École Polytechnique Fédérale de Lausanne (EPFL) in Lausanne, Switzerland [13].

Table 1. List of produced prototypes of microBPMs and their main parameters.

\begin{tabular}{|c|c|c|c|c|}
\hline N. & $\begin{array}{c}\text { Test } \\
\text { Name }\end{array}$ & Substrate & $\begin{array}{l}\text { N. of } \\
\text { layers }\end{array}$ & $\begin{array}{c}\text { Al } \\
\text { thickness }\end{array}$ \\
\hline 1 & $\begin{array}{l}\text { Si-1x- } \\
\text { 8um }\end{array}$ & $\mathrm{Si}-\mathrm{SiO}_{2}$ & 1 & $8 \mu \mathrm{m}$ \\
\hline 2 & $\begin{array}{l}\text { Si-1X- } \\
400 n m\end{array}$ & $\mathrm{Si}-\mathrm{SiO}_{2}$ & 1 & $400 \mathrm{~nm}$ \\
\hline 3 & $\begin{array}{l}\text { Si-1x- } \\
5 \text { Onm }\end{array}$ & $\mathrm{Si}^{-\mathrm{SiO}_{2}}$ & 1 & $50 \mathrm{~nm}$ \\
\hline 4 & $\begin{array}{l}\text { Si-2x- } \\
400 n m \\
\end{array}$ & $\mathrm{Si}^{-\mathrm{SiO}_{2}}$ & 2 & $400 \mathrm{~nm}$ \\
\hline 5 & $\begin{array}{l}\text { PI-1X- } \\
400 n m\end{array}$ & $\begin{array}{l}\mathrm{Si}_{-} \mathrm{SiO}_{2} \text {, coated with } \\
10 \mu \mathrm{m} \text { of polyimide }\end{array}$ & 1 & $400 \mathrm{~nm}$ \\
\hline
\end{tabular}

A first batch of devices was fabricated with the main goal of proving the feasibility of the BPM detectors with the new technique. Table 1 lists the different stacks that were produced and tested. The substrate of all the productions was a $525 \mu \mathrm{m}$ thick 100 mm $\varnothing$ silicon wafer covered with a $1 \mu \mathrm{m}$ thick $\mathrm{SiO}_{2}$ layer (obtained by wet oxidation). In order to confirm that the metal layer thickness does not impact the SEY generation, BPMs featuring three different metal thicknesses were produced. In addition, one device with a second metal layer was produced to try to assess the gain in signal obtained from stacking several sensing layers. Finally, some of the wafers were coated with a $10 \mu \mathrm{m}$ thick polyimide layer before the Aluminum sputtering, in order to evaluate the quality of the Aluminium-polyimide interface. In order to further reduce the total material budget of the detector, the Silicon substrate could be etched away in a last fabrication step and, thus, only the polyimide layer would remain as substrate.

The process flow adopted for the fabrication of each microBPM is described in the following:
- $\quad$ Si-1x-8um was fabricated by sputtering 8 $\mu \mathrm{m}$ of $\mathrm{Al}$ on the $\mathrm{Si}-\mathrm{SiO} 2$ substrate. A photolithography step after the deposition was carried out to produce the desired pads layout.

- $\mathrm{Si}-1 \mathrm{x}-400 n m$ was fabricated by the same process, changing only the thickness of the Al layer.

- Si-1X-50nm fabrication followed similar steps to the previous ones. In addition, for this production, a final $\mathrm{SiO}_{2}$ passivation layer was added on top, to protect the $\mathrm{Al}$ traces between the pads and the contact pads. This $\mathrm{SiO}_{2}$ layer was first sputtered (400 nm thick) and then patterned (by photolithography and subsequent etching) to open vias at the contact positions.

- $\mathrm{Si-2x-400nm}$ was fabricated by $400 n \mathrm{~nm}$ sputtering on the substrate, then deposition of 200nm $\mathrm{SiO}_{2}$ and finally another 40onm $\mathrm{Al}$ layer. The intermediate $\mathrm{SiO}_{2}$ layer was patterned with trenches along the signal traces so as the second metal sputtering step would result in the connection of the two metal layers (Fig. 3).

- PI-1x-40onm was fabricated by spin coating of a $10 \mu \mathrm{m}$ thick polyimide layer on the substrate and then following the same process performed for $\mathrm{Si}-1 \mathrm{x}-400 \mathrm{~nm}$.

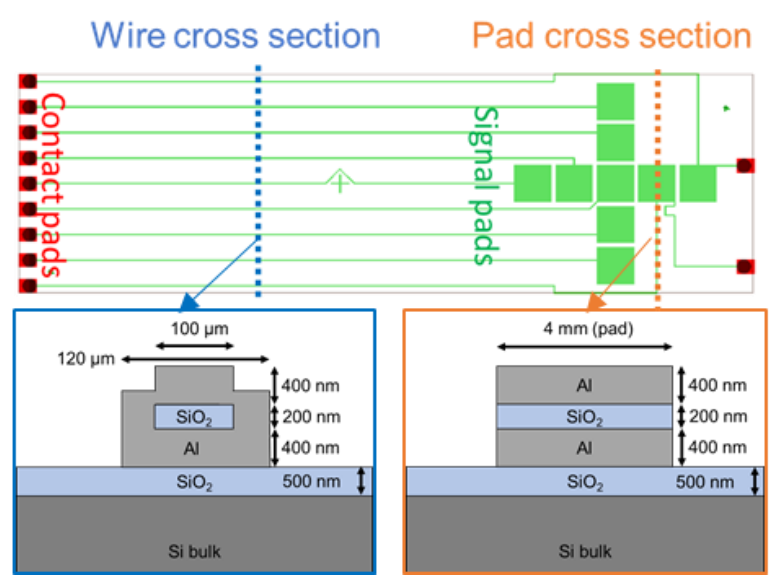

Figure 3. Cross section of a trace taken along the blue line in (left), and cross section of a pad along the orange line (right), for the Si-2x-400nm microBPM.

\section{EXPERIMENTS}

The micro-BPMs were mounted in the center position of one of the IRRAD tables (Fig. 4.a), on the existing sample-holder, and connected to the readout system in the control room through a patch-panel. A mini-BPM was placed back to back with the new micro-BPMs allowing a direct comparison of the electrical signal measured from each BPM while being crossed by the same proton spill. The signal collected by the pads was acquired and treated by the BPM data acquisition system shown in Fig. 4.b. The BPM readout uses low noise integrators through which the charge 
generated on the pad after the crossing of particles flows. The developed voltages from each integrator are then processed by an Arduino-Yun microcontroller and the data is stored on an online database ready for visualization [3].
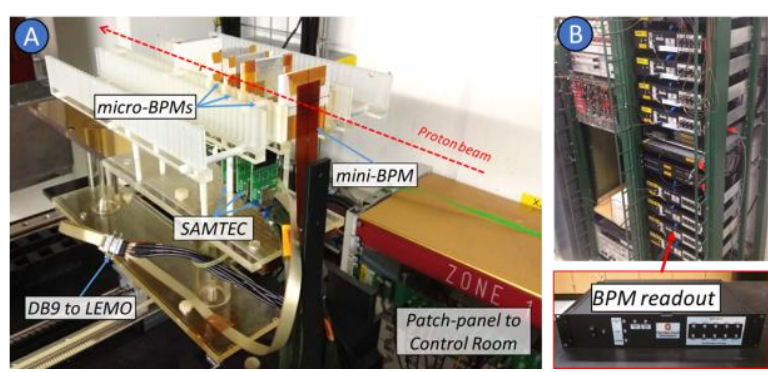

Figure 4. (a) Micro-BPMs with DB9 and SAMTEC connectors, installed on the irradiation table in IRRAD, together with a reference mini-BPM. (b) Rack of BPM data acquisition system located in the IRRAD control room connected to the inside of the IRRAD bunker through shielded $25 \mathrm{~m}$ long micro-coaxial cables.

\section{RESULTS AND DISCUSSION}

Due to the short time available for production and testing before the end of the 2018 irradiation run, most of the devices were installed in the facility without any prior electrical testing. Moreover, many difficulties were encountered to connect the devices to the readout system. Since direct soldering on the contact pads was unsuccessful, some devices were wire-bonded to an intermediate $\mathrm{PCB}$, used to interface with the same type of connector as the standard mini-BPMs; for some other devices, the cable to the readout box was directly connected to the pads using silver glue. As a consequence of these issues, the results of the experiments were not satisfactory for most of the tested devices. In many of them, significant differences in signal level were observed among the different pads, with some channels showing very small signal while, for others, the voltages given by the integrators saturated the $\mathrm{ADC}$ even for very low intensity beams. Faulty connections or defects in the fabrication are most likely responsible for these effects.

The best results were obtained for a device with a $400 \mathrm{~nm}$ sensing layer on $\mathrm{Si}$ (process number 2 in Table 1), so we focus here on the outcome of this test. Comparing qualitatively the beam profiles measured by the mini-BPM with the ones of the micro-BPM positioned back-to-back (Fig. 5) on the on-line BPM display, a very good resemblance is clearly visible for both $\mathrm{X}$ and $\mathrm{Y}$ directions (three consecutive spills are shown in the figure).

In order to study the linearity in the response of the device with respect to the particle flux, the beam intensity during the test was lowered, in five steps, from $\sim 8 \cdot 10^{11} \mathrm{p} / \mathrm{cm}^{2}$ to $\sim 10^{11} \mathrm{p} / \mathrm{cm}^{2}$.

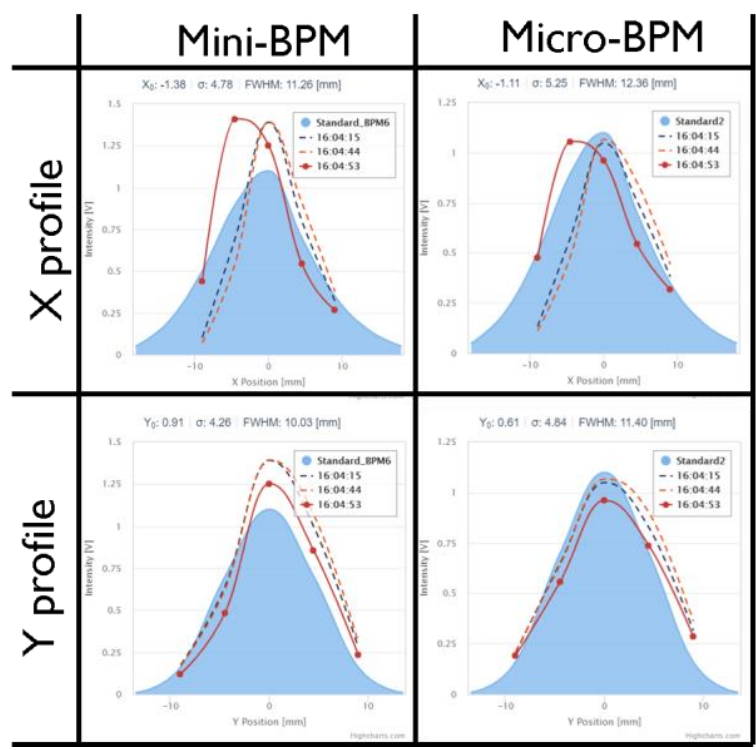

Figure 5. Comparison between the profiles of three different proton spills measured with a mini-BPM and a micro-BPM (Si-1x-40onm). Red line corresponds to the current spill, while dashed lines correspond to the previous proton spill (orange) and one before (black). The blue, filled area in the background represents the expected profile with standard beam conditions.

Figure 6 shows a scatter plot of the voltages measured by the micro-BPM vs the ones measured by the mini-BPM, for all spills during the intensity scan. The different colors represent the 9 pads of the cross pattern. The signal level, while slightly lower for the micro-BPM remains comparable to the mini-BPM. All micro-BPM pads show a similar correlation with their mini-BPM counterparts, with some variance which could be attributed to the imperfect alignment of the 2 detectors, and the focusing or defocusing of the beam from one device to the other. The slightly sub-linear characteristic between the two devices must be attributed to the micro-BPM, since the mini-BPM response is known to be linear in this range of operation. Measurements with a Vector Network Analyzer were performed on the cables connecting the micro-BPM with the readout unit to rule out interference between channels that could be responsible for this nonlinear behavior. No cross-talk was observed, which lead us to believe that the observed effect could be attributed to exchange of electrons between pads, liberated due to the interaction with the proton beam.

Figures 7 and 8 show a comparison between the accumulated horizontal, respectively vertical, profiles measured by both devices for the whole duration of the experiment. Even though the measurements from the two devices are, qualitatively, in good agreement, the profiles measured by the micro-BPM are slightly broader than the ones measured by the mini-BPM. This effect can be explained by the sub-linear response discussed in the previous paragraph: the higher signal intensity at the beam center is more attenuated than at the tails, thus leading to a flattening of the observed profile. 


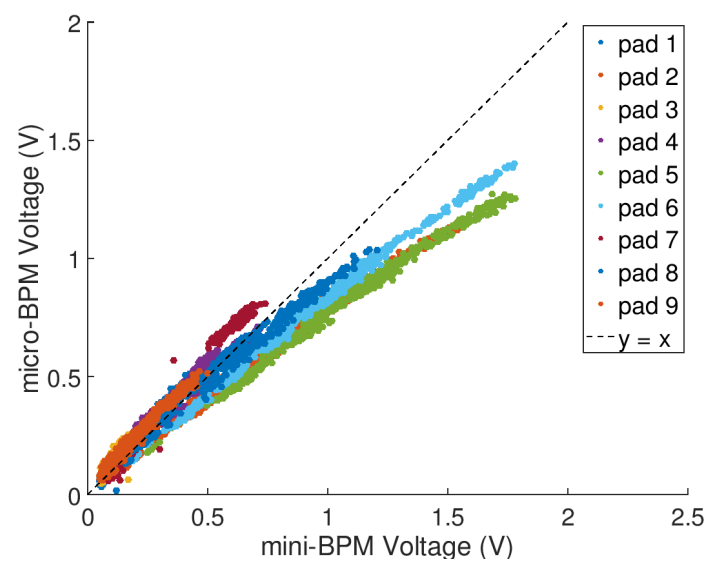

Figure 6. Scatter plot of the voltage measured by a $400 \mathrm{~nm} \mathrm{Al}$ on Si micro-BPM vs the voltage measured by an old miniBPM installed back to back, during a beam intensity scan from $8 \cdot 10^{11} \mathrm{p} / \mathrm{cm}^{2}$ to $\sim 10^{11} \mathrm{p} / \mathrm{cm}^{2}$. Different colors represent the different pads of the cross-shaped devices.

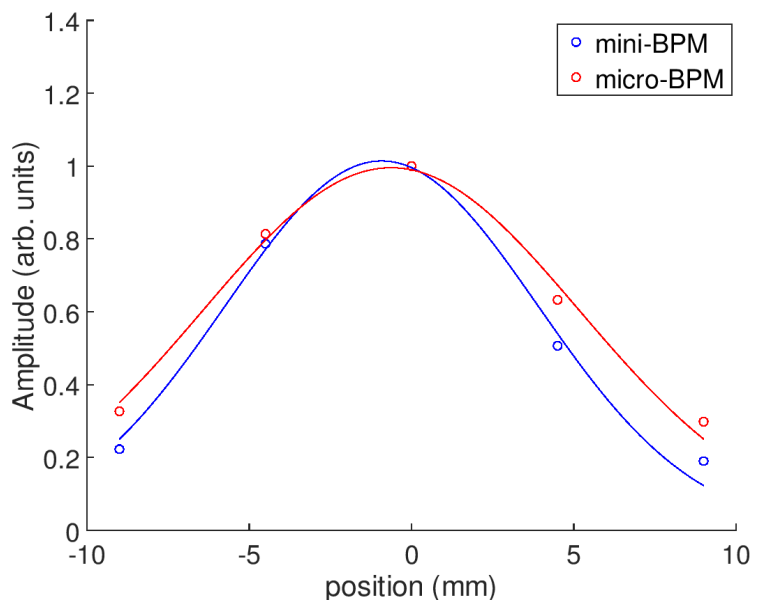

Figure 7. Comparison of the accumulated horizontal profiles measured by the mini-BPM (blue) and micro-BPM (red), during a beam intensity scan from $\sim 8 \cdot 10^{11} \mathrm{p} / \mathrm{cm}^{2}$ to $\sim 10^{11} \mathrm{p} / \mathrm{cm}^{2}$.

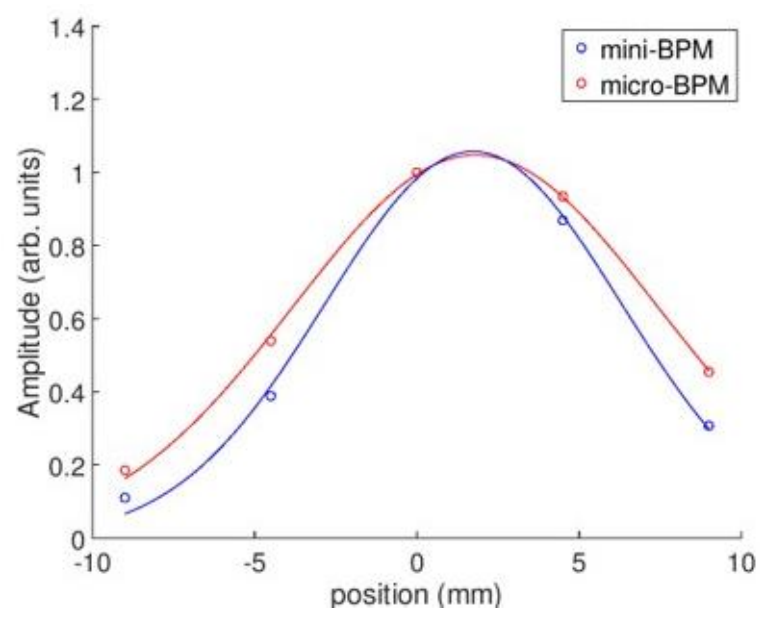

Figure 8. Comparison of the accumulated vertical profiles measured by the mini-BPM (blue) and micro-BPM (red), during a beam intensity scan from $\sim 8 \cdot 10^{11} \mathrm{p} / \mathrm{cm}^{2}$ to $10^{11} \mathrm{p} / \mathrm{cm}^{2}$.

\section{CONCLUSION}

The deposition of thin ( $<1 \mathrm{um})$ metal layers on different substrates, using state-of-the-art microfabrication techniques is being investigated as a novel approach for the fabrication of Beam Profile Monitors for the IRRAD facility at CERN. The main benefits of this technique are lower material budget (hence, reduced interaction volume), higher radiation resistance and higher design flexibility.

Several devices were produced varying the number of metal layers and the metal thickness, in order to try to disentangle the impact of these parameters on the device response. However, this was not possible since, due to the low yield in the fabrication and the lack of reliable connections to the readout electronics, many of the tested devices did not produce measurable results.

Despite the encountered difficulties and the shortage of beam time available, a fully working prototype, featuring a $400 \mathrm{~nm}$ Aluminum layer on a Silicon substrate, could be tested. The comparison of the profiles measured by this device versus the ones obtained by the old mini-BPM have been presented in this article and constitute the proof of principle to carry on future developments.

Further tests are needed to fully characterise the response of the new devices. Mainly, two effects need to be investigated: the sub-linear characteristic of the device signal versus the beam intensity, and the apparent broadening of the beam profile measured with the new micro-BPM, when compared to the ones measured with the old mini-BPM. To do so, a new production of BPM's is currently ongoing, and tests in new irradiation facilities are being considered for testing during CERN Long Shutdown 2, in which the IRRAD facility is not operational. One such facility is CLEAR [14], at CERN, where the micro-BPM are being characterized under a $200 \mathrm{MeV}$ electron beam [15].

Since the experimental results on the first tests showed comparable signal levels between the old miniBPM and the new BPM with only one metal layer, the new production will focus only on single layer devices, thus simplifying the production. Devices with different metal thickness will be again produced, in order to establish the comparison that was not possible in this work. In addition, since some electron exchange between pads seemed to occur on the tested devices, an attempt will be made to produce BPM detectors on polyimide substrates, instead of Silicon, with the idea of improving the isolation between channels and ruling out any contribution of the Silicon substrate on the measured signal. To achieve this, the deposition of Aluminium on polyimide, which was already tried during the first production but did not result in any fully working prototype, will need to be further investigated. Replacing the Silicon substrate with a less dense material would have the additional benefit of extending the range of application of the system to beams of lower energy.

Acknowledgement: The authors wish to thank the staff of the Center of Micronanotechnology (CMi) of the École Polytechnique Fédérale de Lausanne (EPFL) for providing their assistance during cleanroom production. In addition, we would like to express our 
D. Bouvet et al., Low-mass radiation-hard beam profile monitors..., RAD Conf. Proc., vol. 5, 2021, 9-14

thanks to Beam Instrumentation (BI) group for useful discussion about secondary emission theory and all the operators of the CERN-PS machine.

This project has received funding from the European Union's Horizon 2020 Research and Innovation programme under Grant Agreement no. 654168.

\section{REFERENCES}

1. B. Gkotse et al., "A New High-intensity Proton Irradiation Facility at the CERN PS East Area," in Proc. PoS TIPP2014, Amsterdam, Netherlands, 2014, article no. 354.

https://doi.org/10.22323/1.213.0354

2. F. Ravotti, et al., "The Beam Profile Monitoring System for the CERN IRRAD Proton Facility" in Proc. $5^{\text {th }}$ International Beam Instrumentation Conference (IBIC), Barcelona, Spain, 2016, pp. 825-828 https://doi.org/10.18429/JACoW-IBIC2016-WEPG75

3. H. Seiler, "Secondary electron emission in the scanning electron microscope", J. Appl. Phys., vol. 54, no. 11, pp. R1 -R18, 1983 https://doi.org/10.1063/1.332840

4. B. Gkotse, M. Glaser, E. Matli, F. Ravotti, "System architecture and data processing capabilities of the Beam Profile Monitor for the CERN IRRAD Facility", presented at the IEEE Nuclear Science Symposium Conf. (NSS/MIC/RTSD), Strasbourg, France, 2016. https://doi.org/10.1109/NSSMIC.2016.8069891

5. C. Cuccagna et al., "Beam parameters optimization and characterization for a Turning LInac for Protontherapy", Physica Medica, vol. 54, pp $152-156$, Oct 2018 https://doi.org/10.1016/j.ejmp.2018.08.019

6. V. Agoritsas, "Secondary emission chambers for monitoring the CERN Proton Synchrotron ejected beams", presented at Daresbury Symposium on Beam Intensity Measurement, Daresbury, England, 1968. Retrieved from:

http://cds.cern.ch/record/299104/files/CERN-MPSInt-co-68-9.pdf Retrieved on: February 13, 2020

7. S. Weisz, "A luminosity monitor for the LHC", Ph.D. dissertation, University of Lausanne, Lausanne, Switzerland, 2001. Retrieved from: https://cds.cern.ch/record/508769/files/thesis-2001013.pdf

Retrieved on: February 13, 2020

8. F. Roncarolo et al., "Wire grid and wire scanner design for the CERN LINAC4", in Proc. of Linear Accelerator Conference (LINAC2010), Tsukuba, Japan, 2010, pp. 650-652.

Retrieved from:

https://cds.cern.ch/record/1303302/files/tup101.pdf

Retrieved on: February 13, 2020

9. Koyama, T. Shikata, H. Sakairi, "Secondary Electron Emission from $\mathrm{Al}, \mathrm{Cu}, \mathrm{Ag}$ and $\mathrm{Au}$ Metal Targets under Proton Bombardment”, Jpn. J. Appl. Phys., vol. 20, no. 1, pp. 65-70, 1981.

https://doi.org/10.1143/jjap.20.65

10. E. J. Sternglass, "Theory of Secondary Electron Emission by High-Speed Ions", Phys. Rev., vol. 108, no. 1, pp. 1-12, 1957. https://doi.org/10.1103/PhysRev.108.1

11. T. Koshikawa, R. Shimizu, "A Monte Carlo calculation of low-energy secondary electron emission from metals", J. Phys. D: Appl. Phys., vol. 7, no. 9, pp. $1303-1315,1974$. https://doi.org/10.1088/0022-3727/7/9/318

12. V. Baglin et al., "The secondary electron yield of technical materials and its variation with surface treatments", in Proc. $7^{\text {th }}$ European Particle Accelerator Conference (EPAC 200o), Vienna, Austria, 2000. Retrieved from: https://accelconf.web.cern.ch/eoo/PAPERS/THXF102. pdf

Retrieved on: February 13, 2020

13. Center of MicroNanoTechnology (CMi), EPFL.

Retrieved from:

https://cmi.epfl.ch/

Retrieved on: February 13, 2020

14. CERN Linear Electron Accelerator for Research (CLEAR), CERN.

Retrieved from:

https://clear.cern/clear

Retrieved on: February 13, 2020

15. D. Bouvet et al, "NanoRadMet: Development of MultiPurpose, Low Mass, Beam Profile Monitors by Nanometric Metal Films Deposition.”, Submitted to International Conference on Technology and Instrumentation for Particle Physics (TIPP), Vancouver, 2020. 